\title{
Lab demonstration of the hybrid Doppler wind lidar (HDWL) transceiver
}

\author{
Catherine T. Marx ${ }^{1}$, Bruce Gentry ${ }^{1}$, Patrick Jordan ${ }^{1}$, Peter Dogoda ${ }^{2}$, Ed Faust ${ }^{3}$, Michael Kavaya ${ }^{4}$ \\ ${ }^{1}$ NASA/Goddard Space Flight Center, Greenbelt, Maryland 20771 \\ ${ }^{2}$ Sigma Space, NASA/Goddard Space Flight Center/614.4 \\ ${ }^{3}$ SGT, Inc., NASA/Goddard Space Flight Center/540.0 \\ ${ }^{4}$ NASA/Langley Research Center, Hampton, VA 23681
}

\begin{abstract}
The recommended design approach for the 3D Tropospheric Winds mission is a hybrid Doppler lidar which combines the best elements of both a coherent aerosol Doppler lidar operating at $2 \mu \mathrm{m}$ and a direct detection molecular Doppler lidar operating at $0.355 \mu \mathrm{m}$. In support of the mission, we built a novel, compact, light-weighted multi-field of view transceiver where multiple telescopes are used to cover the required four fields of view. A small mechanism sequentially selects both the "transmit" and "receive" fields of view. The four fields are combined to stimulate both the $0.355 \mu \mathrm{m}$ receiver and the $2 \mu \mathrm{m}$ receiver. This version is scaled ( $0.2 \mathrm{~m}$ diameter aperture) from the space-based version but still demonstrates the feasibility of the hybrid approach. The primary mirrors were conventionally lightweighted and coated with dielectric, high reflectivity coatings with high laser damage thresholds at both $2 \mu \mathrm{m}$ and $0.355 \mu \mathrm{m}$. The mechanical structure and mounts were fabricated from composites to achieve dimensional stability while significantly reducing the mass. In the laboratory, we demonstrated the system level functionality at $0.355 \mu \mathrm{m}$ and at $2 \mu \mathrm{m}$, raising the Technology Readiness Level (TRL) from 2 to 4.
\end{abstract}

Keywords: 3D Tropospheric Winds, Lidar,

\section{SCIENCE JUSTIFICATION}

Direct measurements of the global wind fields will be extremely valuable for numerical weather prediction, as well as scientific diagnostics of large-scale atmospheric transport, weather systems, and boundary layer dynamics in Earth's atmosphere (Stoffelen et al; 2005). At present, the vertical structure of the horizontal wind field is difficult to measure accurately, particularly over the oceans and in the free troposphere. Except for the unevenly distributed single-level measurements at the surface by buoys, aircraft and cruise ships and the very few profile measurements from radiosonde stations, direct wind profile measures are lacking. Satellite-based cloud drift inferred wind measurements are available but have fairly large errors in the wind measurement itself and the validity of height allocation of the measurements is suspect (Cress and Wergen, 2001).

The Global Tropospheric 3D Winds mission has been specifically identified by the recent NRC Decadal Survey report as one of 15 priority missions for Earth Science in the next decade. The weather panel concluded that a Global Wind Mission "will improve acquisition of global observations of wind profiles for assimilation into the latest numerical weather forecast models. There will be substantial benefits from this mission in the form of improved weather forecasts and severe storm predictions. There is also great potential for discovery in the seasonal and inter-annual measurements of winds, aerosols, and clouds from the tropics to the Polar Regions. Wind information will have direct benefits to human safety, downwind of hazardous sources of gases or aerosols. It will also benefit the long-term atmospheric studies associated with climate change.”

The Weather Panel report further recommends that NASA "Design, develop and demonstrate a prototype Hybrid Doppler Wind Lidar (HDWL) system capable of global wind measurements to meet demonstration requirements that are somewhat reduced from operational threshold requirements. All of the critical technologies such as laser, receiver, detector, and control, will be tested in the demonstration HDWL mission. Space demonstration of a prototype HDWL in LEO is to take place as early as 2016.” Our proposal builds and demonstrates the technology necessary for the HDWL system. 


\section{THE MEASUREMENT}

Direct measurement of global vertical wind profiles can be obtained using a Doppler lidar in a polar low earth orbit. Doppler lidars measure winds by collecting the signal backscattered from atmospheric aerosol particles and/or molecules. Figure 1 shows the Doppler shift of spectra for molecular and aerosol backscatter (for different transmitted wavelengths).

A Doppler lidar measures vertical profiles of the component of the wind velocity projected along the line of sight of the laser. Pulsed lasers are used to provide range information based on the time delay of the transmitted pulse. Wind profiles are derived from measurements of backscattered light from atmospheric aerosol particles, and/or molecules generated from an initial laser pulse and measuring the change in frequency from the initial laser pulse. Sensitive frequency measuring techniques are used that are capable of resolving the small Doppler frequency shift induced on the outgoing beam, by the motion of the atmosphere. Because Doppler lidars measure the component of the wind along the line-of-sight of the lidar, at least two observations from different pointing angles are required to resolve the horizontal winds in a sample volume. Figure 2 shows a simplified two-field point measurement concept for obtaining 2-d wind vectors from space. As

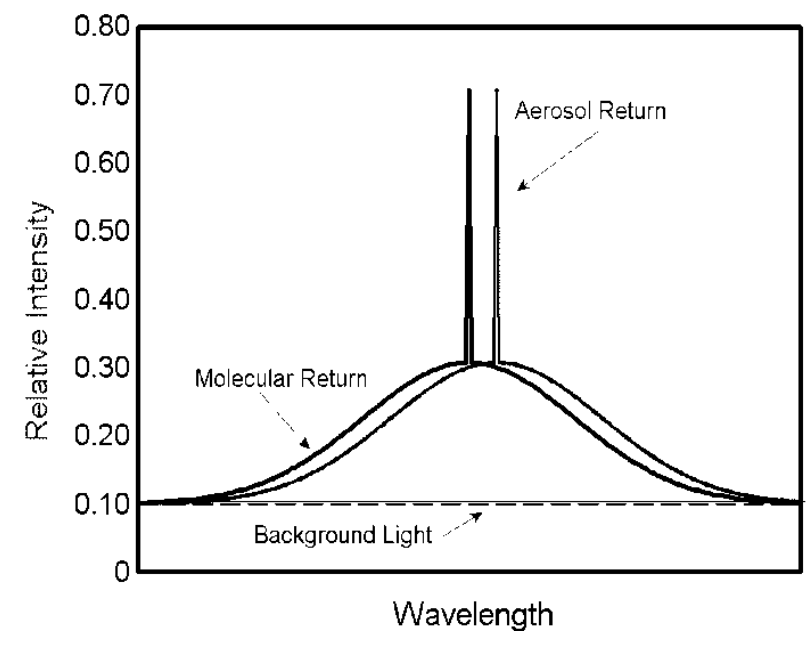

Figure 1: The hybrid system allows detection of the 3D winds from the backscattered return from both molecules and aerosols. the spacecraft translates in orbit, a lidar beam is consecutively directed at a target sample volume from two different perspectives. The lidar has a fixed nadir angle in order to resolve the horizontal component of the wind field. The lasers are alternatively directed fore and aft of the spacecraft to obtain the two perspectives required to sample the wind vector (e.g "u, v horizontal components" or "speed and direction") information. At the location of each target sample volume, backscattered return from rangegated cells in the troposphere is averaged over several laser pulses to reduce measurement noise. Multiple lines of measurements are made across the satellite's ground track. Tradeoffs between dwell time and precision can be made.

At shorter wavelengths in the visible and UV, molecular scattering makes up a large part of the total scattering. At longer wavelengths in the infrared (IR), aerosol scatter dominates. Doppler lidars optimized for aerosol backscatter typically operate in the IR and use coherent detection techniques (Huffaker and Hardesty 1996) which are extremely sensitive for measuring the narrow line width aerosol return. Coherent Doppler lidars have been operated for more than three decades to measure 3-d wind fields in studies from a variety of terrestrial, ship and aircraft platforms. Coherent wind lidar systems do not measure molecular backscatter return. Relatively small satellite-deployed coherent detection lidars could make measurements from high aerosol regions such as the planetary boundary layer and from elevated cloud layers which presently cover over $70-80 \%$ of the globe.

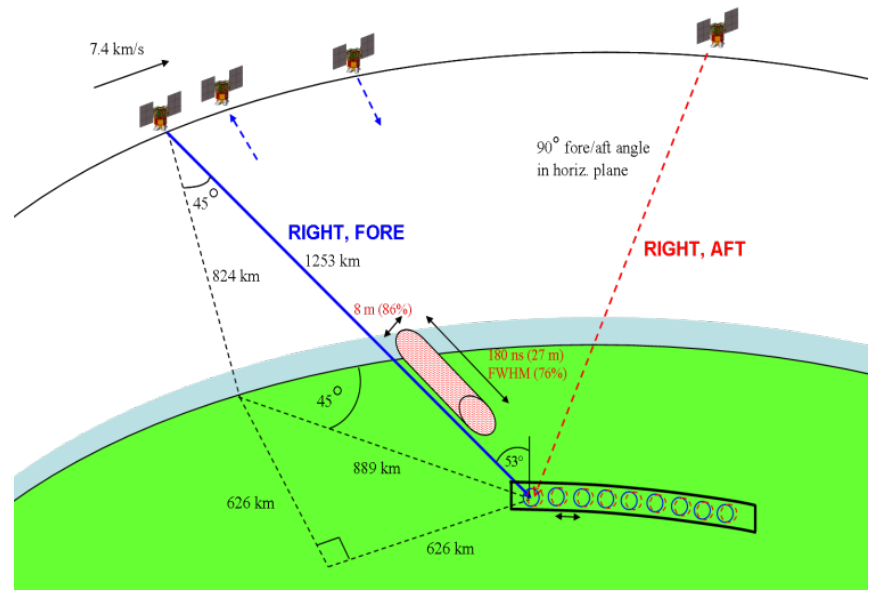

Figure 2: Measurements of horizontal winds are made possible with our space based lidar wind measurement concept.

More recently, direct detection Doppler lidars have been demonstrated (Gentry et al 2000, Gentry and Chen 2002) employing interferometers to measure winds from both molecular and aerosol return. Direct detection Doppler lidars can measure wind velocity when atmospheric aerosol loading is minimal or absent. A satellite direct detection lidar would most likely operate in the UV spectral region where molecular backscatter is highest. However, the molecular backscatter bandwidth is large (Figure 1) so that direct 
detection lidars must collect large numbers of backscattered photons to meet wind accuracy requirements. Thus, high power lasers (tens of watts) and large collecting apertures are needed in space systems. Photon counts can also be improved by increasing collection time or range gate length, both of which degrade spatial resolution.

System studies have investigated both direct and coherent detection Doppler wind lidars that could individually provide the full vertical coverage required. Both types of systems, if designed to provide full wind profiles, would require extremely large lasers and telescopes. For example, the European Atmospheric Dynamics Mission (ADM) (Stoffelen et al. 2005), scheduled for launch in 2010, is a UV direct detection Doppler lidar. A powerful laser (15 watts in the UV) and large telescope (1.5 m diameter) consume so much of the instrument weight and power budget that scanning has been dropped and now only a single fixed (line of sight) perspective will be sampled. ADM provides one example of the tradeoffs faced in designing the instrument, where the trade space typically involves laser power, telescope size, single vs. bi-perspective wind measurement, as well as horizontal and vertical resolution.

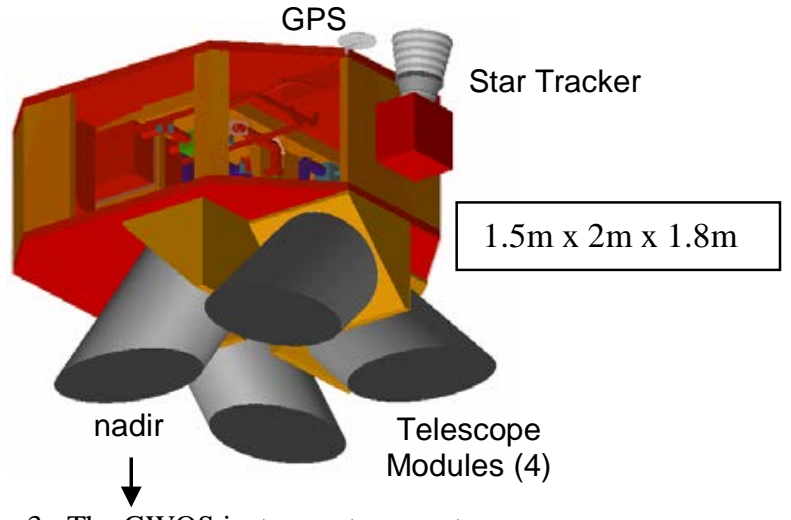

Figure 3: The GWOS instrument concept.

In 2006, NASA HQ supported a hybrid Doppler wind lidar mission study as part of its Advanced Mission Concept Study in preparation for the Decadal survey. The hybrid Doppler wind lidar incorporates both direct and coherent detection techniques. The system architecture was studied in the GSFC Instrument Design Lab as the Global Winds Observing Sounder (GWOS) study. This study was for a prototype HDWL system capable of global wind measurements to meet demonstration requirements that are somewhat reduced from operational threshold requirements. Although ADM data will greatly benefit both instrument development and scientific efforts, the scientific impact will be greatly enhanced (Riishojgaard et al. 2006) when two perspectives of the wind are obtained, as shown in Figure 2. For the GWOS study, bi-perspective sampling of two lines (four looks) across each $350 \mathrm{~km}$ segment of the satellite ground track was assumed. Each look would be sampled for $12 \mathrm{~s}$, providing a local measurement over a sample volume of about 84 $\mathrm{km}$ per line of sight wind measurement. It was packaged to be launched on a Delta II with 0.5 $\mathrm{m}$ apertures for each of the 4 fields of view. Figure 3 shows the GWOS instrument.

In obtaining two perspectives the GWOS concept differs from ADM in two important ways. First, rather than have a single large fixed pointing telescope, four small telescopes, $0.5 \mathrm{~m}$ diameter, are employed oriented with a fixed nadir angle of 45 degrees and with each telescope pointed to one of the corners of a

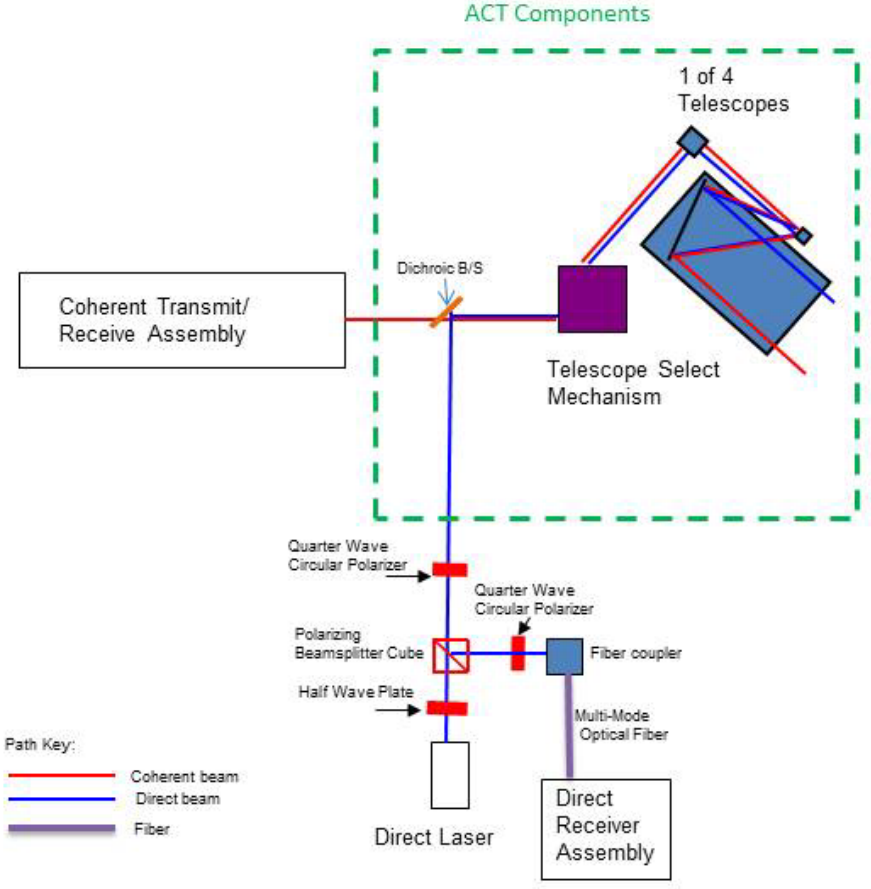

Figure 4: Schematic of ACT hardware. square. The diagonal of the square is rotated by 45 deg relative to the spacecraft direction of motion. Second, the GWOS design calls for a hybrid Doppler lidar approach, incorporating both direct and coherent detection, which makes the best use of limited spacecraft resources. The hybrid approach provides an efficient use of spacecraft resources to obtain a high-impact science product. 


\section{SYSTEM LEVEL REQUIREMENTS}

We designed and built a scaled down version of a shared transceiver optical system that meets the above mission requirements for four lines of sight and multiwavelength operation. The common transceiver simultaneously meets the performance requirements of the coherent Doppler lidar (e.g. diffraction limited field of view with high throughput at 2 microns) and those of the direct detection Doppler lidar (low scatter, high damage threshold coatings with high throughput in the ultraviolet). Figure 4 shows the schematic for the hardware built and assembled during this project. The telescopes, select mechanism, and aft optics were built and aligned into a transceiver package, as shown in Figure 5.
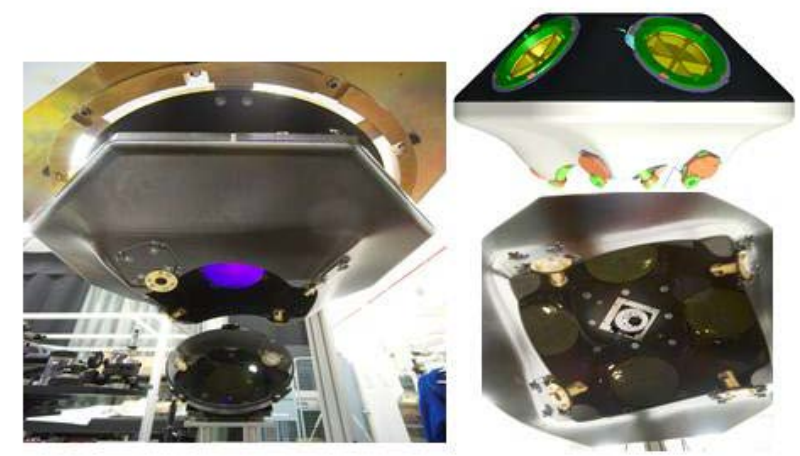

Figure 5: 3D model and pictures of 4-FOV HDWL transceiver

Table 1: First order requirements

\begin{tabular}{|c|c|c|c|c|}
\hline & Lab Demonstration & Lab Demonstration & Space Demo & Space Demo \\
\hline & $0.355 \mu \mathrm{m}$ & $2 \mu \mathrm{m}$ & $0.355 \mu \mathrm{m}$ & $2 \mu \mathrm{m}$ \\
\hline Platform Altitude & $12-20 \mathrm{~km}$ & $12-20 \mathrm{~km}$ & $400 \mathrm{~km}$ & \\
\hline $\begin{array}{l}\text { Telescope Collecting } \\
\text { Aperture }\end{array}$ & $8 ”(0.2 \mathrm{~m})$ & $8 ”(0.2 \mathrm{~m})$ & $0.5 \mathrm{~m}$ & \\
\hline Number of Look Angles & 4 & 4 & 4 & 4 \\
\hline Telescope View Angle & $\begin{array}{c}45^{\circ} \text { above horizon, } \\
\text { equally spaced in } \\
\text { azimuth }\end{array}$ & $\begin{array}{c}45^{\circ} \text { above horizon, } \\
\text { equally spaced in } \\
\text { azimuth }\end{array}$ & $\begin{array}{c}45^{\circ} \text { above horizon, } \\
\text { equally spaced in } \\
\text { azimuth }\end{array}$ & $\begin{array}{c}45^{\circ} \text { above horizon, } \\
\text { equally spaced in } \\
\text { azimuth }\end{array}$ \\
\hline Telescope Magnification & 11.1 & 11.1 & TBD & TBD \\
\hline Telescope Configuration & - & Unobscured telescope & - & $\begin{array}{l}\text { Unobscured } \\
\text { telescope }\end{array}$ \\
\hline $\begin{array}{l}\text { Telescope Throughput } \\
\text { Requirements }\end{array}$ & $>90 \%$ & $>90 \%$ & $>90 \%$ & $>90 \%$ \\
\hline Telescope Image Quality & 95\% in $100 \mu$ rad blur & $\begin{array}{l}\text { Diffraction limited at } \\
\qquad 2 \mu \mathrm{m}\end{array}$ & $\begin{array}{l}95 \% \text { in } 100 \mu \mathrm{rad} \\
\text { blur }\end{array}$ & $\begin{array}{l}\text { Diffraction limited at } \\
22 \mu \mathrm{m}\end{array}$ \\
\hline Field of View & $100 \mu \mathrm{rad}$ & Diffraction limited & $100 \mu \mathrm{rad}$ & Diffraction limited \\
\hline
\end{tabular}

Table 1 First order design requirements. For comparison, the Space Demo (GWOS) requirements are also given. As shown, this lab demonstration is built to specifications similar to those of the Space Demo.

The ESTO Instrument Incubator Program (IIP) has funded development of an airborne $0.355 \mu \mathrm{m}$ system Tropospheric Wind Lidar Technology Experiment (TWiLiTE) and a $2 \mu \mathrm{m}$ airborne Doppler Aerosol WiNd lidar (DAWN). The transceiver developed here is built and designed to operate in an aircraft environment (temperature, pressure, and 
vibration), with the intention that at a later date, the transceiver will be flown with both the TWiLiTE and DAWN IIP lidars to demonstrate the hybrid concept from an airborne platform. Table 2 gives the interface specifications used in the design to accommodate TWiLiTE and DAWN

Table 2: Interface specifications for TWiLiTE and DAWN

\begin{tabular}{|l|l|l|}
\hline & $\mathbf{3 5 4 . 7} \mathbf{~ n m}$ System & $\mathbf{2 . 0 5 4} \mathbf{~} \mathbf{m}$ System \\
\hline & Laser beam input; Fiber feed output & $\begin{array}{l}\text { Common path laser input/output. } \\
\text { Receive beam is split off inside the } \\
\text { VALIDAR trailer }\end{array}$ \\
\hline Pulse Energy & & $250 \mathrm{~mJ}$ \\
\hline Pulse Rep Frequency & $<2.5 \mathrm{~mJ}$ & $10 \mathrm{pps}$ \\
\hline Beam Size & $50 \mathrm{pps}$ & $6 \mathrm{~mm} 1 / \mathrm{e}^{2}$ \\
\hline Polarization & $22 \mathrm{~mm} 1 / \mathrm{e}^{2}$ & Circularly polarized \\
\hline Beam Quality & Circularly polarized & Diffraction limited at $2.054 \mu \mathrm{m}$ \\
\hline Fiber feed for receiver & $\mathrm{M}^{2}<3.5(\sim 80 \mu$ rad divergence) & N/A \\
\hline
\end{tabular}

\section{OPTICS}

A key purpose of the instrument is to house 4 identical telescopes pointing in separate directions as described above. A select mirror is used to select the active telescope. An optics bench houses the common optics for the laser/receiver inputs for the $0.355 \mu \mathrm{m}$ system and the $2 \mu \mathrm{m}$ system. We used the allowable instrument volume in the Global Hawk and an existing aircraft window as constraints for laying out the system. The four telescopes are afocal, off-axis telescopes crossed to reduce the overall volume. The packaging has reduced height and increased width, to accommodate the aircraft space constraints. These constraints led us to such tight packaging requirements that we could no longer accommodate a conventional metering structure for each telescope. The internal volume had to be free of obstructions to accommodate the four beams. An additional packaging constraint included a 21 inch window to for the aircraft. This in turn placed a constraint on the overlap of the four telescope beams at the window. 


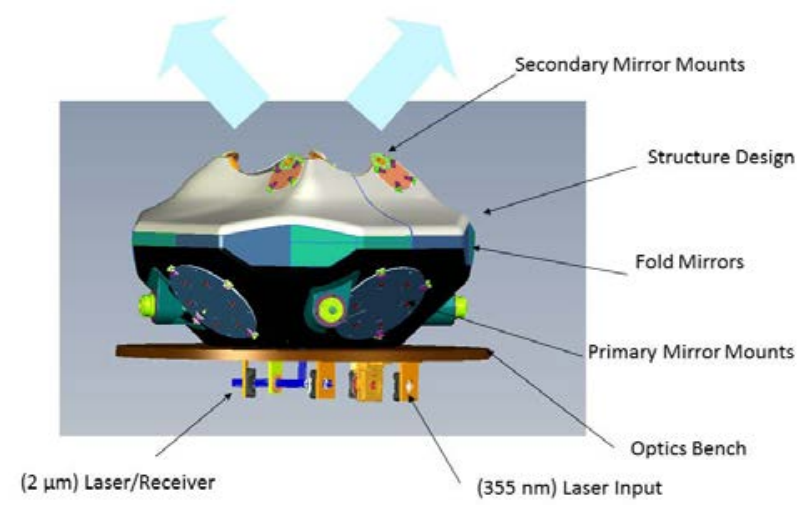

Figure 6: Cut away view of hardware.

Figure 7: CAD view of hardware.

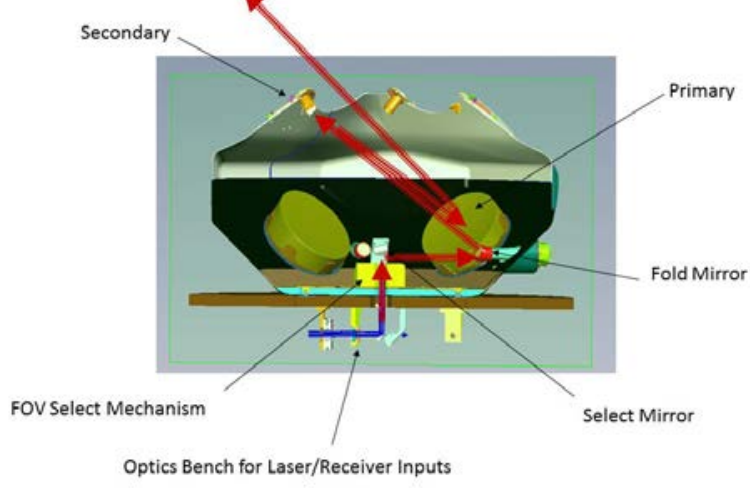

The structure is shown above, both in cut-away view and in full view. The structure holds the telescope optics, fold mirror, select mechanism and its fold mirror. In this upward viewing orientation, the optics bench is below with the optics hanging from it. The laser inputs and receiver outputs interface with the optics on the optics bench. The FOV select mechanism rotates, changing the active telescope and FOV. 


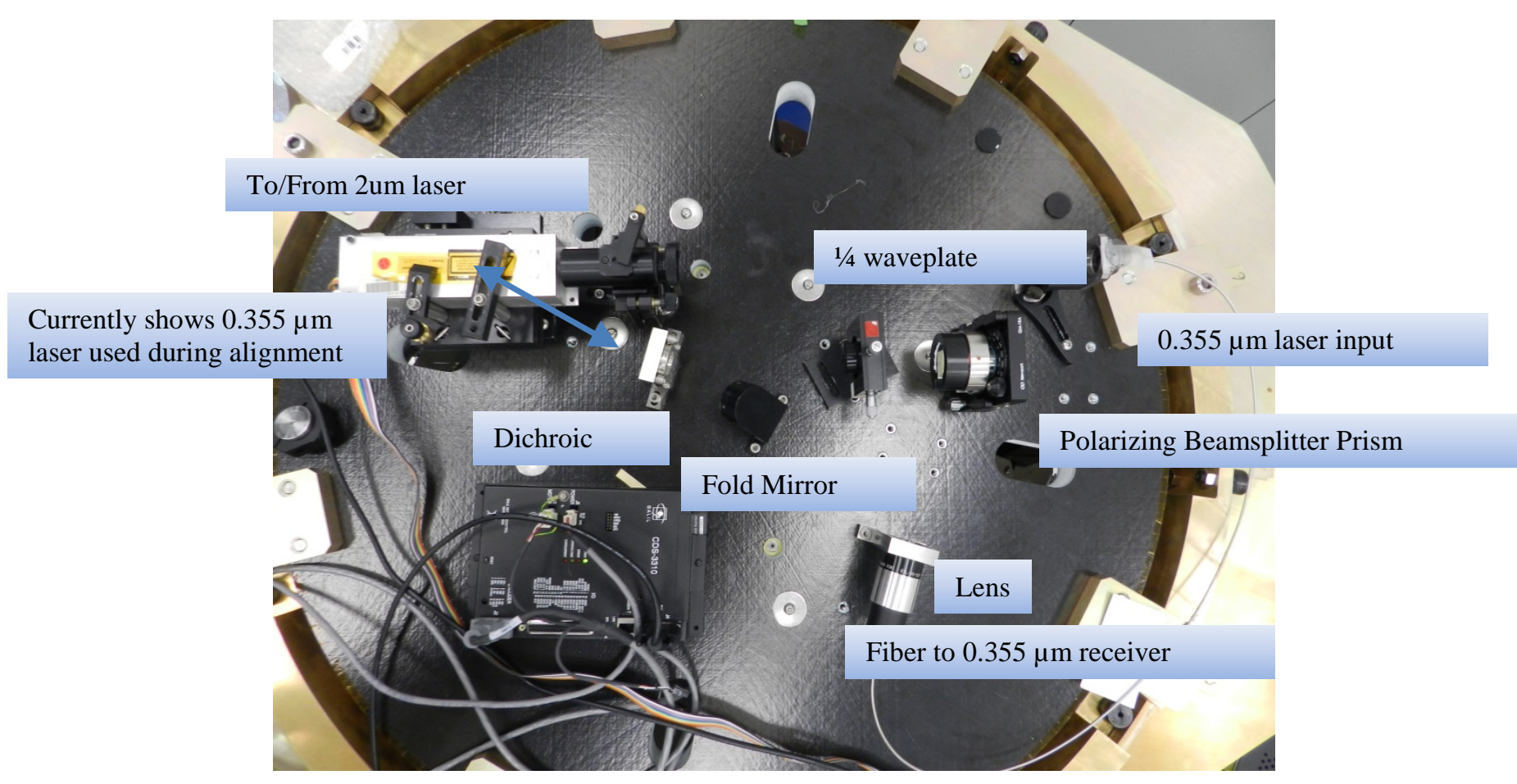

Figure 8: View of optics bench hardware.

Figure 8 Optical bench layout: there is a fold mirror as the beam exits the telescope assembly onto the bench. A dichroic beam splitter transmits the $2 \mu \mathrm{m}$ light and reflects the $0.355 \mu \mathrm{m}$ component. A polarizing beam splitter prism transmits the input $0.355 \mu \mathrm{m}$ polarized laser beam but reflects the return orthogonally polarized beam. A lens focusses the beam on to the $0.355 \mu \mathrm{m}$ receiver fiber.

\section{3. $\quad$ Error Budget}

Two separate error budgets were created for this hardware, one for the $0.355 \mu \mathrm{m}$ system and one for the $2 \mathrm{um}$ system. For the $0.355 \mu \mathrm{m}$ system, the budget is based on RMS spot diameter at the receiver fiber. Basically, the tolerances are based on a throughput/encircled energy requirement as the system needs to maximize the number of photons collected. For the 2 um system, the budget was based on wavefront error. In this case, the coherent system needs to be diffraction limited to maintain acceptable signal-to-noise ratios. The tolerances required for diffraction limited performance at 2 um were the primary design drivers. The most stringent requirements were the alignment between the primary and secondary mirrors. It was the alignment between primary and secondary which led us to use composite materials. With the aim to build to aircraft standards, an aluminum structure was not compatible with the thermal environment. The alignment of the flats and beam splitters did cause some degradation in image quality when the outgoing beam did not follow the telescope optical axis. These flat and beam splitter tolerances were looser than the tolerances between the powered optics. The telescope mirror alignment tolerances are shown in Table 4.

Table 4: Telescope mirror alignment tolerances.

\begin{tabular}{|l|c|c|}
\hline Tip/tilt of Primary & 20 & Arcsec \\
\hline Clocking of Primary & 2 & Arcmin \\
\hline Decenter of Primary & 25 & Microns \\
\hline Focus of Primary & 5 & Microns \\
\hline Tip/tilt of Secondary & 1 & Arcmin \\
\hline Clocking of Secondary & 15 & Arcmin \\
\hline Decenter of Secondary & 25 & Microns \\
\hline Focus of Secondary & 5 & Microns \\
\hline
\end{tabular}


The final error budget (Table 5) requires detailed knowledge of the in-situ environment including vibration and thermal. The fabrication and alignment tolerances were chosen to be tight but achievable and to allow for uncertainties in these unknown categories. We selected the Global Hawk requirements as the structure design criteria, including thermal and vibration environments. The environmental contributions are not included in the table below because we did not design an aircraft compatible bench. As can be seen from this table, the alignment is driven by the $2 \mu \mathrm{m}$ system.

Table 5: Error budget

\begin{tabular}{|l|l|l|}
\hline Contribution & Waves at $\mathbf{2} \boldsymbol{\mu m}$ & RMS Spot Diameter (0.355 $\boldsymbol{\mu m}) \mathbf{( \mu m )}$ \\
\hline Design & 0 & 45 \\
\hline Fabrication & 0.01 & 5 \\
\hline Alignment & 0.057 & 12 \\
\hline Mechanism & 0 & 0 \\
\hline Environmental - Thermal & TBD & TBD \\
\hline Environmental - Handling & TBD & TBD \\
\hline \hline Total (not including environmental) & 0.058 & 62 \\
\hline \hline Requirement & $<0.07$ & $<150$ \\
\hline
\end{tabular}

4.4. Throughput

\subsubsection{High Reflectivity Coatings}

Rainbow Research Optics, Inc. in Centennial, CO provided all the reflective mirror coatings. Several separate coating runs were required. We were looking for high reflectivity at our two wavelengths and the ability to withstand illumination from our two high power lasers (specifications in Table 2). For all samples, the reflectivity was greater than $97 \%$ at $0.355 \mu \mathrm{m}$ and greater than $99 \%$ at $2 \mu \mathrm{m}$. Because the system is used in double pass, the system throughput is a function of the reflectivity of the coating raised to the eighth power. A one percent decrease in reflectivity corresponds to an eight percent hit in throughput. Witness samples were laser damage threshold tested at Quantel, Inc. $(0.355 \mu \mathrm{m})$ and at LaRC $(2 \mu \mathrm{m})$. At the start of the project, these coatings were considered to be high risk. Although they were not cheap, we were able to find a few vendors able to supply these coatings. These survived the testing with no damage.

\subsubsection{Dichroic Beam splitter}

Rocky Mountain Instruments Co. (Lafayette, CO) built a custom designed dichroic beam splitter with low polarization sensitivity. The beam splitter reflected the UV and transmitted the IR. The polarization sensitivity specification was derived from the $2 \mu \mathrm{m}$ system. The polarization was specified to be less than $1 \%$ difference between the transmitted "s" and "p" polarization states. These samples were not laser threshold tested. Other vendors had trouble meeting the polarization specification.

\section{MECHANICAL DESIGN}

\subsection{Composites}

The structure housed four afocal telescopes positioned 90 degrees apart in azimuth and all pointing 45 degrees off nadir. In order to minimize the volume, the telescopes were overlapping each other, making a traditional metering structure for each telescope impractical. We initially looked at aluminum designs but discovered that they had required complex geometries, which implied difficulty in maintaining alignment over the temperature ranges expected on the Global Hawk. We decided that with composites, we could build a monocoque structure which would be light weight, stiff, thermally stable and have far fewer elements. The Goddard Composite Materials Engineering Technology (GoCoMET) team helped to design, analyze and manufacture the monocoque structure. The fabrication and design of this complex shaped monocoque structure did require the development of new technology and design techniques for the composites group.

The MTM-45 material selected is an out of autoclave (OoA) cured composite material with a 180F processing temperature. The low cure temperature enabled the use of a high density foam tool to form the composite shape. Two foam tools were easily manufactured. Eight to twelve layers of laminated carbon-fiber epoxy were formed over the tools and cured at the lower 180F temperature. The top half of the structure is shown in Fig. 9. This foam tool enabled 
a $5 \mathrm{x}$ cost savings over the cost of a metallic tool due to saved production time and reduced material costs. The two pieces were epoxied together with simple interface pieces to create the monocoque structure (Fig. 10).

Had the structure been fabricated from aluminum; the weight estimate would have been at least $33 \%$ heavier than from composites.

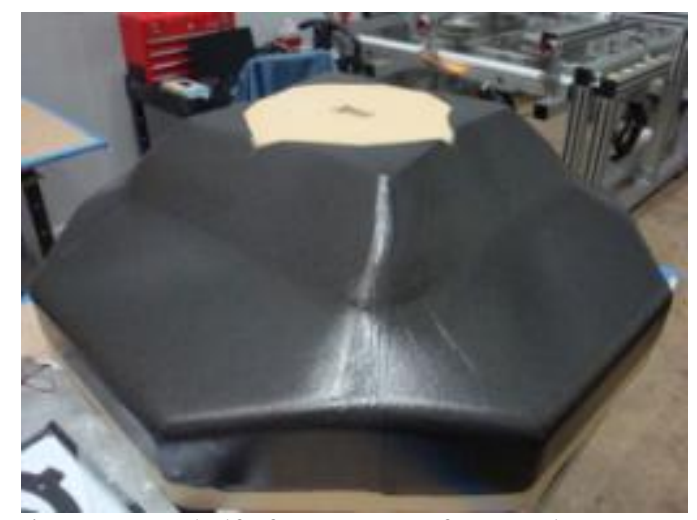

Figure 9: Top half of structure on foam tool

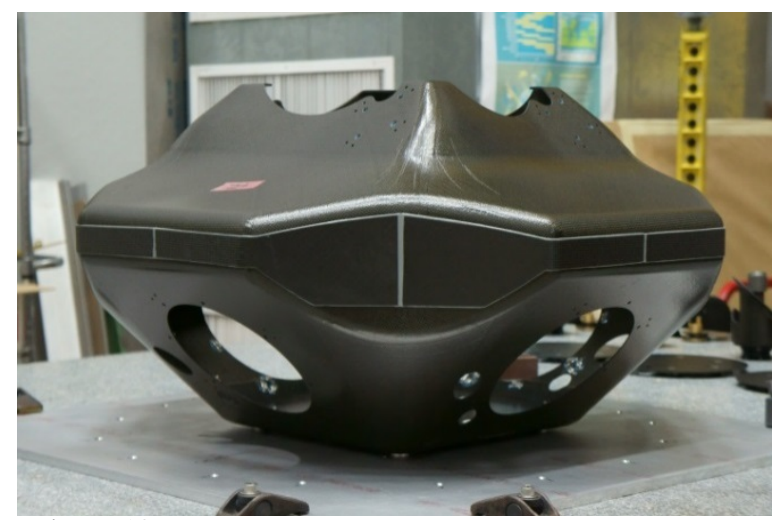

Figure 10: Monocoque structure

\subsection{Mechanical Mounts}

Originally, the mirror mounts were designed from aluminum, but following a thermal analysis, it was determined that the mounts needed to be made from composite materials. The position tolerances on the mirrors did not allow the thermal mismatch between aluminum mounts and the composite structure.

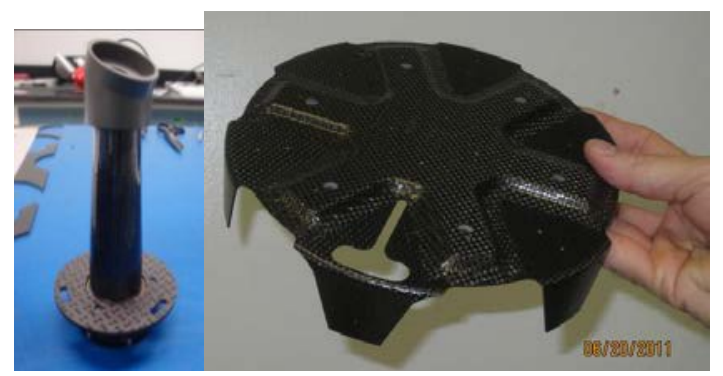

Fig 11: Composite mirror mounts. Fold mirror on left; primary mirror mount on right.

Just as it would be done in metal, the primary mirror mount is a flexure mount with six tabs around the diameter of the mirror. The alignment and orientation of the composite fibers facilitates compliance in the desired axes. The mirror was bonded into these tabs. An interface plate between the mirror and mounting structure was created using a coordinate measuring machine (CMM) to create the correct position of the mirror. Fine clocking, tip-tilt and $x-y$ adjustment of the mirror were completed between the plate and structure. The smaller mirrors were simply bonded into their mounts. Again, all mounts had custom interface plates to facilitate aligning the optics in their nominal positions, and each had some fine adjustment capability. The secondary included tip/tilt and focus adjustment. The fold mirror included $x-y$ and focus. Because the fold mirror was cantilevered, it also had some unintended tip/tilt.

\subsection{Select Mechanism}

This is a step and stare mechanism with a duty cycle of $9 \times 10^{6}$ moves for a 3 year mission lifetime. Each move is assumed to take 1 second to move and settle and 10 seconds to stare in one position. The mechanism will always move in the same

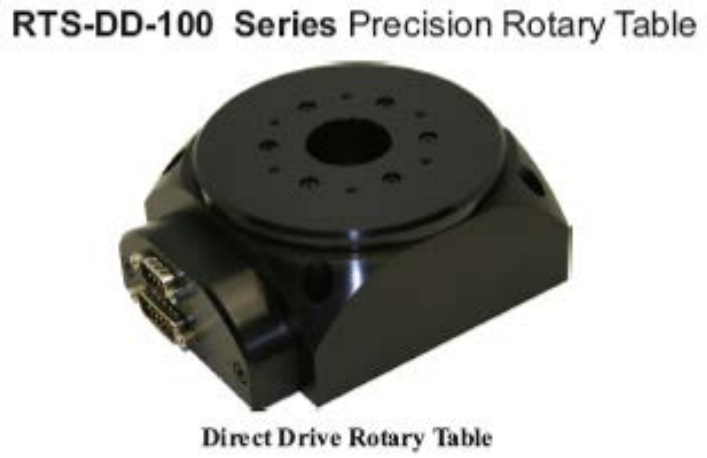

Figure 12: Griffen Rotary Table 
direction. No particular position is preferred if there is a mechanism failure. The Griffen RTS-DD-100 Precision Rotary Table was found to meet the requirements. This mechanism can be made with space flight lube but we used an off-the-shelf version. It performed well in laboratory tests.

\begin{tabular}{|l|l|}
\hline Resolution & 0.32 arc sec \\
\hline Accuracy Full Travel & 30 arc sec \\
\hline Repeatability & 3.0 arc sec \\
\hline Max Velocity & 10 rev sec \\
\hline Run out & 1.5 microns \\
\hline Temp range & -40 to85 C \\
\hline Environmental. pressure & Global Hawk reqts \\
\hline Vibration Data & Global Hawk reqts \\
\hline Voltage & 24 \\
\hline
\end{tabular}

The Galil CDS-3310 Motion Controller with ICM-3300 interconnect module was purchased for use with the select mechanism. The index mechanism harness was routed through the optical bench to the controller. A flight qualified controller can be purchased to control the mechanism.

\subsection{Optics Bench}

Unlike the structure, the optics bench was not designed to aircraft specifications. It was assumed that when these telescopes are flown, a new bench will be built with the appropriate optics and dimensions. Off-the-shelf optics and mounts were built where possible. The bench itself was fabricated from aluminum honeycomb with composite face sheets. The bench is shown in Figure 8.

\section{SUBSYSTEM ASSEMBLY AND PERFORMANCE}

The first steps in the optical assembly of the instrument included bonding mirrors to their mounts and then assembling the mounts into the structure. The interfaces for the mounts had been carefully controlled during fabrication using a CMM. The alignment between the primary and secondary mirrors was critical to the overall alignment of the system. It was the most sensitive alignment and set the pointing direction for each telescope. During fabrication of the mirrors, the backs were polished to be perpendicular to the optical axis and fiducials were scribed onto the back surface indicating the direction to the parent vertex. Using auto-collimators, the two mirrors were set such that their backs were parallel to each other. Very little adjustment was needed. The fold mirrors were placed in their nominal positions.

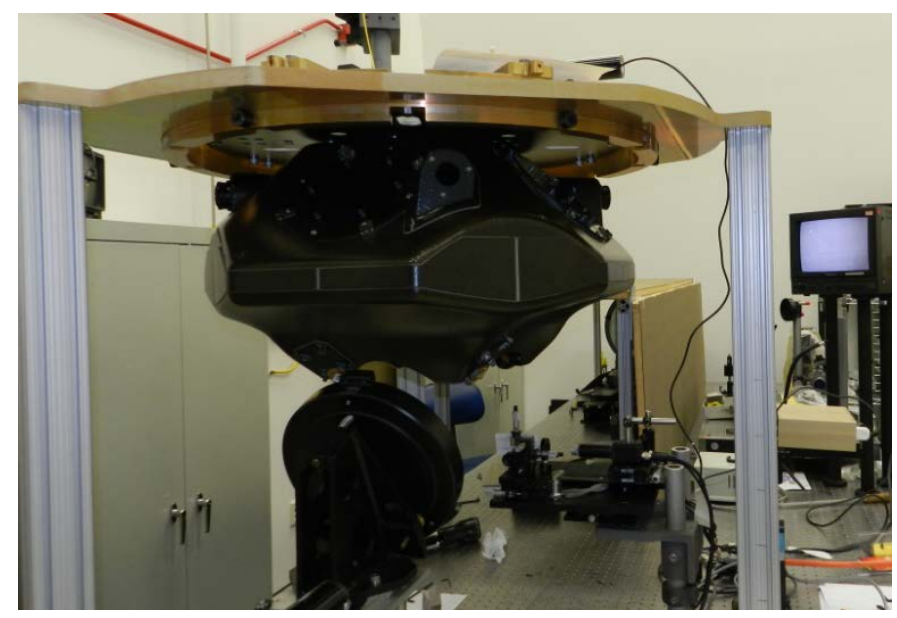

Figure 13: Structure in front of collimator. Large lazy Susan allows rotates to illuminate any of the four telescopes.

The telescope assembly was placed in front of a large collimator and fold which illuminated the telescope at the proper angle. The entire structure was mounted in a large lazy Susan and could be rotated in an equal but opposite direction to the select mirror so that each of the four telescopes optical paths could be aligned and measured. In practice,, during much of the alignment and testing, the system was illuminated through a reverse optical path, using the receive 
fiber as the source. Images were measured at the focal plane of the collimator. The beam from the receive fiber was collimated to the proper size and folded into the select mirror. The telescope mirrors including the fold, secondary and primary mirrors were tweaked into place ensuring that the beam was centered on the optics and the image quality at the collimator focus was good. The lazy Susan was rotated to the four different positions and the select mirror was similarly rotated to the proper position depending on the telescope in use. This ensured a common path of all four telescopes on the optics bench.

\subsection{Image Quality}

The image quality is best assessed by projecting laser light from a near point source, in this case, a single mode fiber through the optical system and recording the focused spot at the collimator focal plane. Spot images from telescope 1 is shown in Figures 14. We did not have access to a camera with sensitivity at $2 \mu \mathrm{m}$ and so were not able to repeat this measurement directly in the infrared. However, we note that the diffraction limited wavefront requirement will be 5.8 times less stringent at $2 \mu \mathrm{m}$ than at $0.355 \mu \mathrm{m}$. The size of the Airy disk at $2.054 \mu \mathrm{m}$ is shown also in the image for reference. Images were taken in May 2011 after initial alignment of the four telescopes and once again in September after final alignment and lock down of the primary and secondary mirror adjustments. The images for telescope 1 and 2 remained good throughout this procedure. The images for telescopes 3 and 4 degraded between May and September. During this time, all shims were replaced with metal shims and adjustments were locked into place. The fold mirror focus adjustment was found to have quite a bit of play in it so it was cranked to one end of travel and shimmed into place.

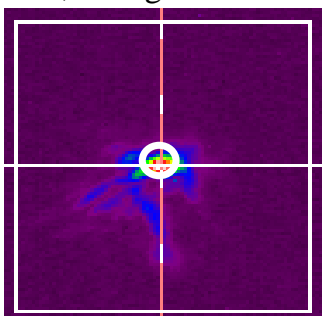

Figure 14: Image from telescope 1 . White circle corresponds to Airy disk at $2 \mu \mathrm{m}$. The adjustment of these telescopes ( 3 and 4 ) needs to be re-evaluated for optimal use in the future.

The boresite was verified by illuminating the system in the "use" direction. A laser was placed at the collimator focus and the beam footprint covered the clear-aperture of the primary mirror. The receive fiber was replaced with a camera and the co-alignment of the each telescope was verified.

\subsection{Throughput}

The end-to-end throughput was measured at both operating wavelengths, $0.355 \mu \mathrm{m}$ and $2 \mu \mathrm{m}$. In both cases, a laser beam of the appropriate wavelength was fed into the optics bench. The beam intensity was measured just prior to the fold mirror on the optics bench. The intensity was again measured after the beam had travelled through the telescopes and to the focus of the alignment collimator. The test was not a full aperture test and so does not include potential losses from vignetting. For the $0.355 \mu \mathrm{m}$ case, the intensity was also measured prior to and after the two alignment mirrors, the large fold flat and off-axis parabola. The measured reflectivity of these two mirrors was subtracted from the final result. We assumed a reflectivity of aluminum of $96 \%$ at $2.054 \mu \mathrm{m}$. Only one of the four telescopes was measured at $2 \mu \mathrm{m}$. The measured results matched our expected values.

\section{CONCLUSION AND FUTURE PLANS}

As part of the ESTO Advanced Component Technology Program, we built a novel, compact, light weighted multi-field of view transceiver to demonstrate a new concept that meets the objectives of the hybrid approach for a future 3D Tropospheric Winds mission. Multiple telescopes are used to cover the required field of view. A small mechanism that sequentially selects both the laser transmit and receive field of view. The transceiver was optimized for performance at the coherent aerosol Doppler lidar wavelength of $2 \mu \mathrm{m}$ and the direct detection molecular Doppler lidar wavelength of $0.355 \mu \mathrm{m}$. The multiple telescope optical assembly is mounted in a unique monocoque composite structure that is both rigid structurally and dimensionally stable in varying environmental conditions. We demonstrated the high reflectivity, high laser damage threshold coating and the mechanism required. At the conclusion of the ACT program we demonstrated the system level functionality at $0.355 \mu \mathrm{m}$ and at $2 \mu \mathrm{m}$, raising the TRL from 2 to 4 .

The prototype telescope has successfully met the ACT goals and objectives in testing in a laboratory. The next step will be to extend the program beyond the laboratory environment to include first a controlled environmental (temperature and vibration) test program and then a real world system level test of the transceiver in one, or both, of the ground based Doppler lidar testbeds developed at Goddard (GLOW/direct detection) or Langley (VALIDAR/coherent). The telescope structure was designed to operate in an airborne environment so an ultimate additional step would include a full airborne demonstration of the Hybrid Doppler lidar concept. 


\section{ACKNOWLEDGEMENTS}

We would like to acknowledge the contributions of the GoCoMET team in the development of the composite structure, especially our primary contacts Kenneth Segal (GSFC/Code 543) and Ronald Glenn (GSFC/Code 540/Jackson and Tull). The design and development of the Hybrid transceiver was supported by the Advanced Components Technology program of the Earth Science Technology Office (ESTO). Additional support from the Goddard Internal Research And Development (IRAD) program is also gratefully acknowledged.

\section{REFERENCES}

[1] Cress, A., and W. Wergen, 2001: Impact of profile observations on the German Weather Service's NWP system. Meteor. Z., 10, 91-101.

[2] Gentry, B. M. et al, 2000: Wind Measurements with A 355 nm Molecular Doppler Lidar, Optics Letters, 25.

[3] Gentry, B. M. and H. Chen 2002: "Performance validation and error analysis for a direct detection molecular Doppler lidar", SPIE Third International Asia-Pacific Environmental Remote Sensing Conference, Hangzhou, China, Oct 23-27, 2002

[4] Gentry, B. M. et al. 2007: "Development of an airborne molecular direct detection Doppler lidar for tropospheric wind profiling”, Proceedings of the SPIE International Symposium on Optical Science and Technology, San Diego, CA, August 28-30, 2007

[5] Huffaker, R. M. and Hardesty, (1996): Remote Sensing of Atmospheric Wind Velocities Using Solid-State And CO2 Coherent Laser Systems, Proceedings of the IEEE.

[6] Kavaya, M. J., B. M. Gentry, Ramesh K. Kakar, and George J. Komar, “Compact Hybrid Doppler Wind Lidar,” Invited Paper, AIAA SPACE 2007 Conference \& Exposition, Session “Technology for Enhancing Future Space-Based Remote Sensing,” Long Beach, CA (18-20 Sept. 2007)

[7] Riishojgaard, L. P., R. Atlas and G. D. Emmitt, 2006: The Impact of Doppler Wind Observations on a Single-Level Meteorological Analysis, J. Applied Met, 43, 810 - 820.

[8] Stoffelen, A. et al, 2005: The Atmospheric Dynamics Mission for Global Wind Field Measurement, Bulletin of the American Meteorological Society, 86, 73-87, 39 pp.

[9] Strategic Plan for the U.S. Integrated Earth Observing System, 2005: May be accessed at: http://usgeo.gov/docs/EOCStrategic_Plan.pdf. 